\title{
DARK CURRENT AND MULTIPACTING IN THE PHOTOCATHODE RF GUNS AT PITZ*
}

\author{
J. H. Han ${ }^{\dagger}$, J. Bähr, H.-J. Grabosch, M. Krasilnikov, V. Miltchev, A. Oppelt, B. Petrosyan, \\ S. Riemann, L. Staykov, F. Stephan, DESY, D-15738 Zeuthen, Germany \\ M. V. Hartrott, BESSY GmbH, D-12489 Berlin, Germany \\ K. Flöttmann, S. Schreiber, DESY, D-22607 Hamburg, Germany \\ J. Rönsch, Hamburg University, D-22607 Hamburg, Germany \\ P. Michelato, L. Monaco, D. Sertore, INFN Milano-LASA, I-20090 Segrate (MI), Italy
} \begin{abstract}
For photocathode rf guns, the amount of dark current depends on the cavity surface as well as on the photocathodes. Smooth conditioning reduces the amount of dark current. Multipacting in the gun cavity changes the surface status of the cathodes and sometimes obstructs the gun operation because of vacuum interlocks. In this paper, dark current and multipacting features of an rf gun are presented including experimental and simulation studies.
\end{abstract}

\section{INTRODUCTION}

Since the VUV FEL and the European XFEL demand high gradients and long rf pulses at the gun, the amount of dark current is comparable to the beam current and can be a hazard to the downstream components like cryogenic modules and undulators. Most of the dark current is cut by beam pipes and collimators. But, dark current starting close to the center of the cathode can be accelerated together with the electron beam. Therefore, understanding on the source location as well as the dynamics of dark current is crucial to prevent component damage and radiological problems.

In this paper, the dark current behavior of several cathodes and of two gun cavities at different conditioning status are discussed and a critical dark current source is found. In addition to the dark current defined by the FowlerNordheim relation, multipacting occurring at the cathode is discussed.

\section{DARK CURRENT PROPERTIES}

Dark current has been measured with a removable Faraday cup located $78 \mathrm{~cm}$ downstream of the cathode. The signal of dark current has been measured at the end of the rf pulse. The dark current signals were almost constant over the rf pulse during the measurements. Figure 1 shows Fowler-Nordheim plots [1] for different status of the cathodes and the cavities. Until November 2003, the first PITZ gun cavity named prototype \#2 has been used for operation. This gun cavity showed low dark current. At present, this gun cavity is used as the injector of the VUV FEL at Hamburg. When we started to use the second PITZ gun

\footnotetext{
* This work has been partly supported by the European Community contract RII3-CT-2004-506008 and by the 'Impuls- und Vernetzungsfonds' of the Helmholtz Association contract VH-FZ-005.

†jang.hui.han@desy.de
}

cavity named prototype \#1 in January 2004, a large amount of dark current was measured. After several months of operation including conditioning efforts, the cavity showed a dark current level even less than the previous cavity.

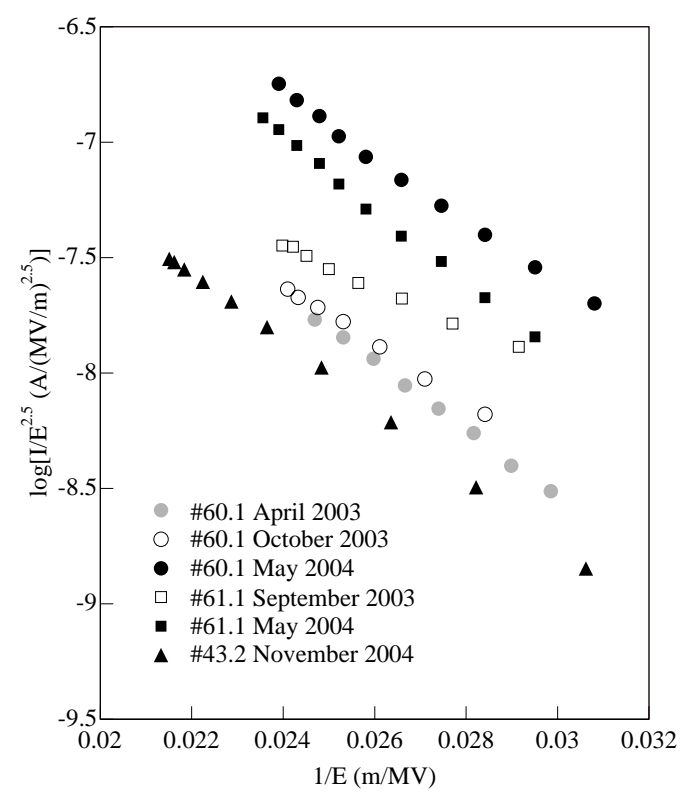

Figure 1: Fowler-Nordheim plots for different status of the cathodes and the cavities. In September 2003 cathode \#61.1 [2] was used the very first time. Cavity \#2 was used until November 2003 and cavity \#1 is being used since January 2004. In May 2004 the $\mathrm{Cs}_{2}$ Te film of cathode \#60.1 was damaged. Cathode \#43.2 was very fresh and well conditioned in November 2004.

Figure 2 displays momentum spectra of dark current and electron beam. The momentum spectra have been measured with a spectrometer dipole $3.45 \mathrm{~m}$ downstream and a YAG screen in a dispersive section. In order to collect a wide range of momentum data, the current of the dipole has been scanned and a series of the projected beam images have been averaged [3].

The high momentum part of the dark current is overlapping with the electron beam to a small extent. According to the simulation results, the overlapping part consists of secondary electrons as well as field-emitted electrons [4]. The secondary electrons can be emitted at an emission phase similar as the electron beam. 

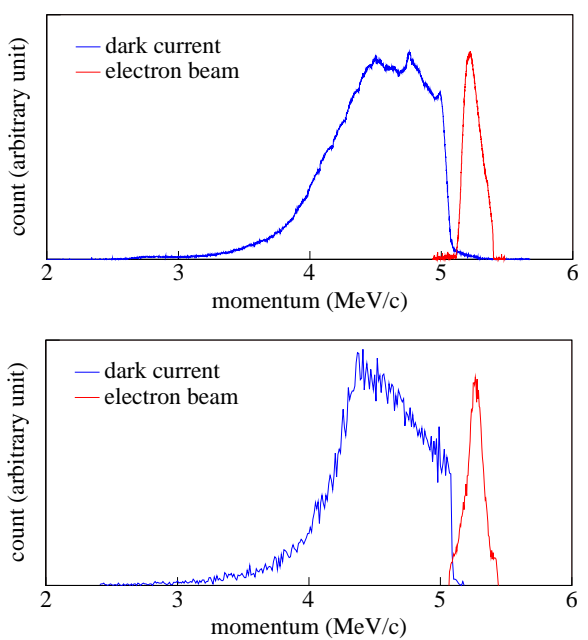

(a)

(b)

Figure 2: Momentum spectra of dark current and electron beam: (a) measurement with a spectrometer dipole and (b) simulation assuming $47 \mathrm{MV} / \mathrm{m}$ maximum field strength at the cathode.

\section{DARK CURRENT SOURCE}

Main dark current sources of the rf gun cavity are the photocathode, the cavity backplane close to the cathode and the two irises because a strong surface field is present at these positions. According to numerical calculations, the field-emitted electrons from the two irises cannot leave the gun cavity but the electrons emitted from the area around the cathode can reach the beamline downstream.

Dark current images are shown in Fig. 3. The images were taken at a screen $0.78 \mathrm{~m}$ downstream from the cathode. Two ring structures are clearly visible at the dark current image without electron beam (Fig. 3b). It can be compared with the cathode geometry (Fig. 3a): The inner ring comes from the boarder of the $\mathrm{Cs}_{2} \mathrm{Te}$ film and the outer ring from the cathode plug edge.

In order to demonstrate this, the emission location of the beam was scanned by moving the drive laser on the cathode. For this measurement, a drive laser with low intensity (electron bunch charge $\sim 2 \mathrm{pC}$ ) and small spot size $\sim 0.5$ $\mathrm{mm}$ rms was used to make the brightness of the beam comparable to that of the dark current and also to make the beam image size smaller. Due to the remarkable QE difference between the $\mathrm{Cs}_{2} \mathrm{Te}$ film $(\gtrsim 1 \%)$ and the Mo plug $(\lesssim 0.01 \%)$, the boundary between $\mathrm{Cs}_{2} \mathrm{Te}$ and Mo could be found (Fig. 3c). The laser spot position on the cathode surface was moved horizontally as well as vertically in $1 \mathrm{~mm}$ steps with a mirror located at the end of the telescope system between the laser source and a view port of the vacuum tube. In the figure, only the images taken for every second step are displayed.

When the whole part of the laser hits the $\mathrm{Cs}_{2} \mathrm{Te}$ area, a bright circular image of the electron beam can be seen. When moving the laser to the boarder, a part of the circular image is getting cut. When the laser spot location was

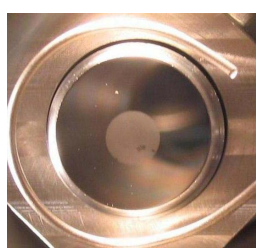

(a)

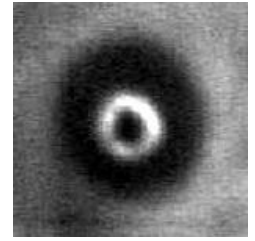

(b)

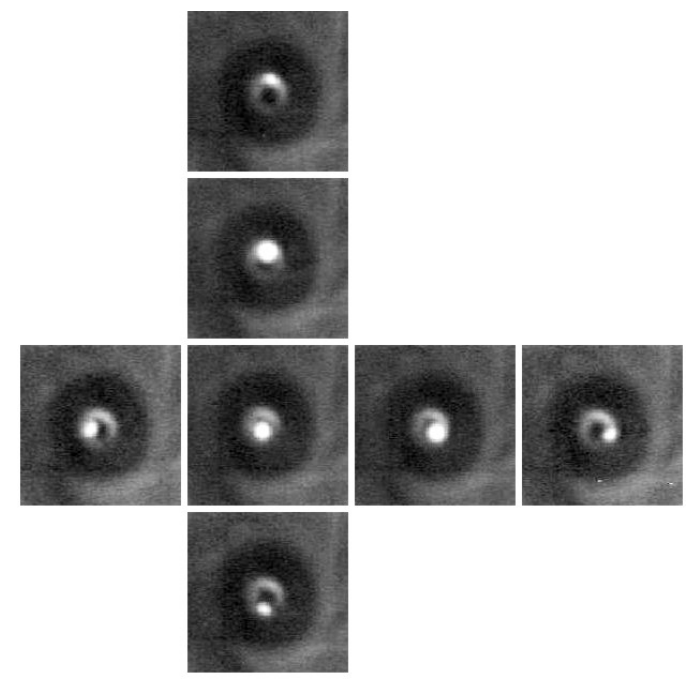

(c)

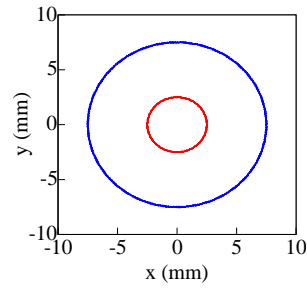

(d)

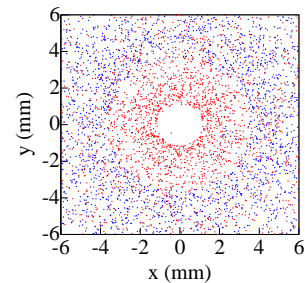

(e)
Figure 3: (a) The front surface of the cathode in the cathode chamber. $\mathrm{A} \mathrm{Cs}_{2} \mathrm{Te}$ film (2.5 mm radius) is coated on a Mo cathode plug ( $8 \mathrm{~mm}$ radius). The wire surrounding the cathode is an anode used for QE measurement of the cathode. (b) Dark current image at a screen $0.78 \mathrm{~m}$ downstream of the cathode. Two ring structures are visible. (c) Electron beam images together with the dark current at the same screen. The emission location of the beam was changed by varying the drive laser location on the cathode by $1 \mathrm{~mm}$ steps. Here only every $2 \mathrm{~mm}$ step is shown. The measurements have been compared with a numerical simulation with ASTRA [5]. (d) Initial distribution of electrons. (e) Simulation result.

moved by one $(1 \mathrm{~mm})$ step further, the beam image completely disappeared.

As described in the previous section, the amount of dark current depends not only on the cathode but also on the cavity status. However, as the electrons propagate downstream only the electrons emitted near the center of the cathode can survive through collimators. Therefore, the inner ring 
produced by the boarder of $\mathrm{Cs}_{2} \mathrm{Te}$ gets important.

\section{MULTIPACTING}

During operation of the gun cavity, two multipacting peaks are found at the beginning and the end of the $\mathrm{rf}$ pulse $[6,7]$. The delay between end of the rf pulse and the rear multipacting peak have been measured for various maximum rf field amplitudes in the cavity (Fig. 4). In March 2004, the dark current in gun cavity \#1 was very high and the photocathodes were damaged quickly due to an insufficient vacuum environment. Cathode \#60.1 was almost completely damaged when the multipacting measurements took place. In September 2004, the gun cavity was well conditioned and the dark current was low as shown in Fig. 1. During a long shutdown of the gun operation between December 2004 and March 2005, the cathode was kept in the cathode chamber under ultra-high vacuum. When gun operation started again, cathode \#43.2 showed a similar behavior as 7 month before.

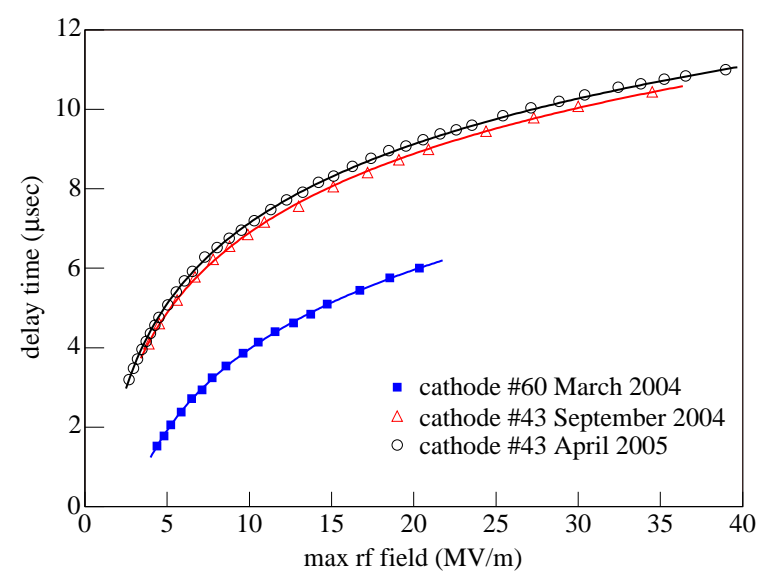

Figure 4: Delay time of the multipacting peak vs. maximum rf field at the cathode. The measurements were made with a main solenoid current of 400 A and a bucking solenoid current of $30 \mathrm{~A}$.

Assuming that the multipacting occurs at the same gradient in the cavity for a cathode and a certain solenoid profile, the multipacting condition can be found with the following relation for the rear peak [7]

$$
E_{\mathrm{MP}}=E_{\max } \exp \left(-t_{\text {delay }} / \tau\right)
$$

where $E_{\mathrm{MP}}$ is the rf field when the multipacting occurs, $E_{\text {max }}$ is the maximum field of the rf pulse at the center of the cathode, $t_{\text {delay }}$ is the delay between the end of the rf pulse and the beginning of the rear multipacting peak, and $\tau$ is the fill/decay time of the rf field in the cavity. In this study, the rear peak is discussed because the time difference between the start of the rf pulse and the first multipacting peak is much less than $1 \mu$ s and hard to measure accurately.

In the actual rf system, the rf field in the cavity is not zero but a certain, very small level when the klystron is running but no low level input is fed into the klystron. Including this background rf term, Eq. 1 can be rewritten as

$$
E_{\mathrm{MP}}=\left(E_{\max }-E_{\mathrm{BG}}\right) \exp \left(-t_{\text {delay }} / \tau\right)+E_{\mathrm{BG}},
$$

where $E_{\mathrm{BG}}$ is the rf background when the low level rf input is not applied. From Eq. 2, the delay time can be expressed as a function of $E_{\max }$,

$$
t_{\text {delay }}=\tau \ln \left(E_{\max }-E_{\mathrm{BG}}\right)-\tau \ln \left(E_{\mathrm{MP}}-E_{\mathrm{BG}}\right) .
$$

For the rf gun operation, the digital signal processing system and the preamplifier of the klystron are adjusted in order to minimize the background level [8].

Three series of measurements for the different status of two cathodes have been performed and the results are shown with fits in Fig. 4. $\chi$-square fits have been made to find $E_{\mathrm{MP}}, E_{\mathrm{BG}}$, and $\tau$ using Eq. 3. The fit results are summarized in Table 1. The two different cathodes show different multipacting conditions $\left(E_{\mathrm{MP}}\right)$. The reason is under investigation. The fitted $\mathrm{rf}$ fill/decay times for three different measurements have a good agreement with 2.78 $\mu$ s obtained from the rf measurements of the cavity.

Table 1: Fit results for the multipacting measurements.

\begin{tabular}{lccc}
\hline \hline cathode & \#60.1 & \multicolumn{2}{c}{ \#43.2 } \\
measured time & Mar. 04 & Sep. 04 & Apr. 05 \\
\hline$E_{\mathrm{MP}}(\mathrm{MV} / \mathrm{m})$ & 2.70 & 1.04 & 1.07 \\
$E_{\mathrm{BG}}(\mathrm{MV} / \mathrm{m})$ & 0.36 & 0.18 & 0.31 \\
$\tau(\mu \mathrm{s})$ & 2.80 & 2.83 & 2.83 \\
\hline \hline
\end{tabular}

\section{DISCUSSION}

If we extend the measurements in Fig 1 for the XFEL gun operating condition $(60 \mathrm{MV} / \mathrm{m})$, the dark current is estimated to be of the order of several $\mathrm{mA}$. Therefore, further studies on dark current are crucial in order to reduce it or to separate it from the electron beam. In order to understand the multipacting condition, empirical and numerical studies are ongoing.

\section{REFERENCES}

[1] J. W. Wang and G. A. Loew, SLAC-PUB-7684, 1997.

[2] Information on the photocathode is available at http://wwwlasa.mi.infn.it/ttfcathodes/.

[3] D. Lipka, PITZ Note 01-04, 2004.

[4] J. H. Han, M. Krasilnikov, and K. Flöttmann, Phys. Rev. ST Accel. Beams 8, 033501 (2005).

[5] K. Flöttmann, A Space Charge Tracking Algorithm (ASTRA), http://www.desy.de/ mpyflo/.

[6] W. Hartung et al., presented at the FNPL Advisory Committee, 2002 (unpublished).

[7] J. H. Han et al., Proc. EPAC 2004, p. 357.

[8] T. Thon and R. Wenndorff (private communication). 